\title{
EFFECTIVE SLIP LENGTH OF NANOSCALE MIXED-SLIP SURFACES
}

\author{
NAT J. LUND ${ }^{1}$ and SHAUN C. HENDY ${ }^{\bowtie 1,2}$
}

(Received 9 June, 2008; revised 23 January, 2009)

\begin{abstract}
We present an approximate relation for the effective slip length for flows over mixed-slip surfaces with patterning at the nanoscale, whose minimum slip length is greater than the pattern length scale.
\end{abstract}

2000 Mathematics subject classification: primary 76N20; secondary 74F10.

Keywords and phrases: Navier slip boundary condition, effective slip.

\section{Introduction}

The central task of fluid mechanics is to find a velocity flow field that satisfies the Navier-Stokes equations and appropriate boundary conditions. The standard boundary condition for flow over a solid surface is no slip. That is, fluid is at rest at the boundary; it does not slip along the wall. With only the odd exception, experiments in the late nineteenth century by Couette and others appeared to verify the no-slip condition. However, the pioneers of fluid mechanics recognized that there was no a priori reason to assume no slip, so the possibility of slip was considered. In fact, in 1823 Navier proposed the boundary condition

$$
\left.u\right|_{z=0}=\left.\delta \frac{\partial}{\partial z} u\right|_{z=0},
$$

that is, the velocity at the boundary is proportional to the shear rate at the boundary. The constant of proportionality is the slip length. If the velocity profile is linearly extrapolated into the wall, the slip length is the depth into the wall at which the velocity would be zero (see Figure 1).

The first reliable observation of slip was probably by Schnell in 1956 [19]. More recent experiments confirm slip [3, 5]. In 2001, Craig et al. [8] reported a sheardependent slip length of up to $\sim 20 \mathrm{~nm}$.

\footnotetext{
${ }^{1}$ MacDiarmid Institute for Advanced Materials and Nanotechnology, School of Chemical and Physical Sciences, Victoria, New Zealand.

${ }^{2}$ Industrial Research Limited, Gracefield, Lower Hutt, New Zealand; e-mail: s.hendy@irl.cri.nz.

(C) Australian Mathematical Society 2009, Serial-fee code 1446-1811/2009\$16.00
} 


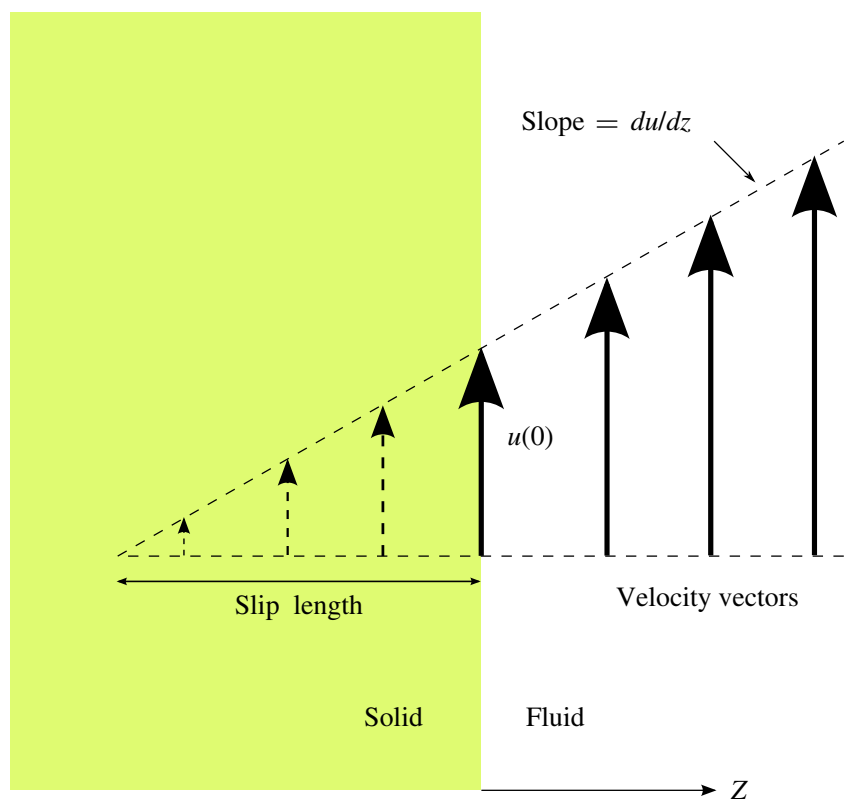

FIGURE 1. Schematic diagram of slip length. Velocity at wall $=$ slip length $\times$ slope and $u(0)=$ $\delta \partial u(0) / \partial z$.

Molecular dynamics simulations have been employed to study slip. In 1990, Thompson and Robbins [20], working with a Lennard-Jones liquid, showed slip in the hydrophobic regime, and epitaxial ordering of the fluid nearest the wall for the hydrophilic regime. Bocquet and Barrat $[1,2]$ report a slip length exceeding 30 atom diameters for a contact angle of $140^{\circ}$.

The physical mechanism of slip is not yet understood. Vinogradova's review article from 1999 [21] provides an excellent overview of slip. In it she identifies three candidate models for slip: (1) molecular slip; (2) existence of a gas layer on the surface; (3) a boundary layer on the surface having very low viscosity. She notes that the experimentally observed slip in thin capillaries [5] could be due to a gas gap only one or two atoms thick, with a corresponding slip length 50 times thicker, that is, 10-20 nm.

Two papers by Zhu and Granick provide insight into the role of roughness in the fluid-solid interaction. In the first, from 2001 [23], experiments with deionized water flow over atomically flat surfaces show the onset of slip above a critical velocity. The slip length varied with velocity, and the onset velocity correlated with contact angle. The second, from 2002 [24], involved flow over similarly hydrophobic surfaces of differing roughness. It was found that roughness suppressed slip, with the no-slip condition holding for roughness above $\sim 6 \mathrm{~nm}$.

In 2002, de Gennes [9] outlined a theory of slip caused by a gas layer at the boundary, noting that the origin of the film remains obscure. However, prior to this, 


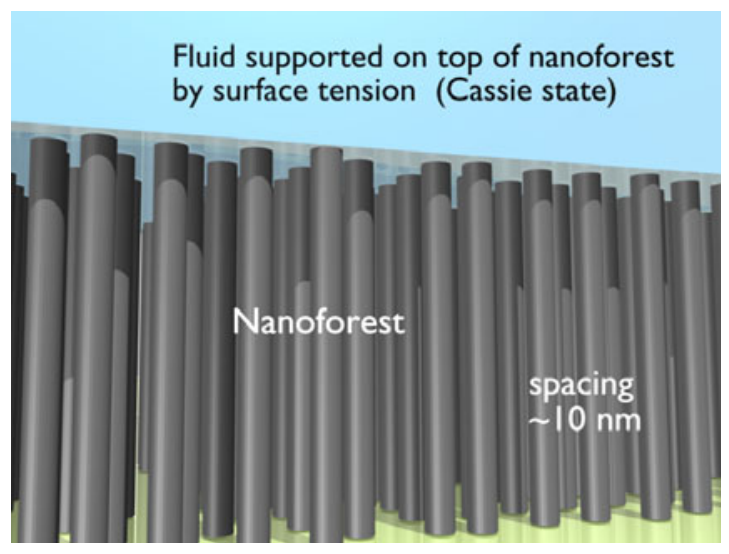

FIGURE 2. Water supported by surface tension on top of a nanoforest.

in 2001, Tyrrell and Attard published a study in which an atomic force microscope was used to discover that a hydrophobic surface was almost completely covered in what appeared to flat nanobubbles of height 20-30 nm. Recently (2008), compelling evidence for nanobubbles on hydrophobic surfaces using infrared spectroscopy has been provided by Zhang et al. [22]. Nanobubbles of air 5-80 $\mathrm{nm}$ high are stable for at least days, but their existence is not necessarily ubiquitous; energy is required for their creation, and their presence depends on the history of the surface.

It is now clear that the measurement of slip length is easily confounded. It is useful to distinguish between the intrinsic slip length of a pure material, and the effective slip length that is actually measured. Measured slip may in fact be for flow over a mixedslip surface, for example, air (nanobubble) and solid substrate. The static properties of such a binary surface have been studied. In 1996 Onda et al. [15] demonstrated a "super-water-repellent fractal surface", on which a water droplet has a contact angle of a remarkable $174^{\circ}$. The droplet is supported by surface tension on top of the hydrophobic dendrites (the Cassie state). This "Fakir" effect of superhydrophobic surfaces (as they are now known), is accompanied by very low resistance to the droplet's motion [17]. Such surfaces occur in the plant kingdom, and are responsible for the self-cleaning properties of the lotus leaf, for example [14]. A general schematic of a superhydrophobic surface is shown in Figure 2. Water is supported by surface tension on top of a hydrophobic nanoforest. With sufficient pressure, the fluid will penetrate the nanoforest down to the substrate (Wenzel state).

Flow over superhydrophobic surfaces was investigated by Cottin-Bizonne et al. in 2003 [7]. Using a molecular dynamics simulation, they showed for the first time that roughness could increase slip length: at sufficiently low pressure, a dewetting transition occurs, and the fluid flows on the top of hydrophobic pillars, in the Cassie state. In 2006 Choi and Kim attempted to fabricate a superhydrophobic surface for maximum slip: teflon-coated silicon nanoturf [4]. They claim a measured slip length of 
20 microns, an order of magnitude greater than slip measured over a carbon nanotube forest by Joseph et al. [12] that same year.

The utility of a relation for effective slip length in terms of intrinsic slip lengths should now be obvious. The first major theoretical assault on the problem seems to be by Philip in 1972 [16], analysing flow over binary surfaces consisting of alternating strips of no-slip and no-shear (infinite slip) conditions. For strips parallel with the direction of flow, the effective slip length is $\delta_{\text {eff }}=(L / \pi) \ln \sec (\phi \pi / 2)$, where $L$ is the period of the striping, and $\phi$ is the area fraction of the infinite slip region. Lauga and Stone in 2003 [13] derive a (numerical) effective slip length for pressuredriven flow down a capillary, whose wall consists of alternating rings of no-slip and no-shear conditions. In a follow-up paper to [7], Cottin-Bizonne et al. advance an argument for the effective slip length of alternating strips of infinite-slip and partialslip boundary conditions [6]. Introducing an interfacial friction coefficient, $\eta / \delta$ (where $\eta$ is viscosity), which is then area averaged, they show that

$$
\delta_{\mathrm{eff}}=\left[\phi \frac{1}{\delta_{s}}+(1-\phi) \frac{1}{\delta_{g}}\right]^{-1},
$$

where $\delta_{s}$ and $\delta_{g}$ are the slip lengths of the solid (partial slip) and gas (infinite-slip) regions.

In a previous paper [11], we have given a more rigorous foundation for result (1.2), in the case of alternating strips of period $L$, where $\delta_{s}>L$. It is the purpose of the present paper to extend this analysis to the case of alternating squares of period $L$.

\section{Method}

The general physical situation consists of fluid flow across a surface of periodically changing slip length. The velocity vector is $\vec{u}=(u, v, w)$, where $u, v, w$ are the velocities in the $x, y, z$ directions, respectively. The fluid (nominally water) has pressure $p$, density $\rho$ and kinematic viscosity $\nu$, and is assumed incompressible, so obeys the continuity equation. Although our analysis is valid for an arbitrarily changing slip length, for clarity we shall often speak of a binary mixed-slip surface, for example water supported by surface tension on the top of a nanoforest (Cassie state).

Our first assumption is that the surface is the flat $x y$-plane $z=0$. We denote the intrinsic slip length of the solid area as $\delta_{s}$ and that of the gas as $\delta_{g}$. The pattern is square-periodic with period length $L$, and the area fraction of the solid portion is denoted $\phi$. The Navier slip condition holds at the surface.

Our second assumption is that the flow is driven by shear, not by pressure. At some height $D$ above the surface is a driving plate moving at steady velocity $u_{D}$. The noslip condition holds at the plate, giving the top boundary condition $u(x, y, D)=u_{D}$. A consequence of the lack of pressure gradient is that the flow field is periodic:

$$
u(x+L, y, z)=u(x, y+L, z)=u(x, y, z) .
$$


Thus, steady-state flow satisfies

$$
\begin{gathered}
(\vec{u} \cdot \nabla) \vec{u}=-\frac{1}{\rho} \nabla p+v \nabla^{2} \vec{u}, \\
\nabla \cdot \vec{u}=0, \\
v(x, y, D)=w(x, y, 0)=w(x, y, D)=0, \\
u(x, y, D)=u_{D}, \\
\delta(x, y) \frac{\partial}{\partial z} u(x, y, 0)=u(x, y, 0) .
\end{gathered}
$$

Consider the slip condition (2.5). If the slip length $\delta(x, y) \rightarrow 0$ across the surface, then the condition becomes the no-slip boundary condition: $u(x, y, 0)=0$. On the other hand, if $\delta(x, y) \rightarrow \infty$, the slip condition $\partial / \partial z(u(x, y, 0))=u(x, y, 0) / \delta(x, y)$ reduces to the shear-free boundary condition $\partial / \partial z(u(x, y, 0))=0$. These two extremes of the slip boundary condition give rise to two exact solutions for sheardriven flow: the no-slip condition results in the Couette flow solution, in which the velocity profile is a linear $z$ gradient. The shear-free (perfect slip) condition results in plug flow, in which the velocity throughout the fluid is identical to the driving velocity. In addition, a shear-driven flow over a surface of uniform slip length will result in a linear velocity profile-essentially Couette flow extrapolated down to the slip length.

We aim to find the effective slip length of a mixed-slip surface. This is the uniform slip length that determines the linear velocity profile of fluid that is above a boundary layer. The periodically changing slip length will cause a periodic flow disturbance, which will die away with increasing height. The height $H$, at which the perturbation has become negligible, is the height of the boundary layer. Obviously, there is some choice in the definition of $H$. By dimensional arguments, $H$ must be a function of $L$ and $\phi$. The velocity at the top of the boundary layer is steady: $u(x, y, H)=u_{s}$.

What conditions hold within the boundary layer? We consider the superhydrophobic regime, wherein the surface consists of high-slip solid regions and near-perfect-slip gaseous regions. If the boundary layer is sufficiently thin, then even the lowest slip velocity will be close to the velocity at the top of the boundary layer. In terms of slip length, $\delta_{\min } \gg H$. Figure 3 illustrates this geometrically.

In other words, flow is close to plug flow. As such, it can be formally expressed as a perturbation of plug flow. A suitable perturbation small parameter expressing the closeness to plug flow is $\epsilon=H / \delta_{\text {min }}$.

Now, $H$ is not a length scale of the physical system; it is emergent. In a 2005 precursor to the present paper [10], it was shown that $H \sim L$. Furthermore, in a nanoscale superhydrophobic regime, with $\delta_{\min } \sim 20 \mathrm{~nm}$, we expect that $\delta_{\min } \gg L$. In that case, our perturbation parameter can be defined in a more fundamental manner, $\epsilon=L / \delta_{\min }$. We present a perturbative analysis of plug flow for the nanoscale superhydrophobic regime. 


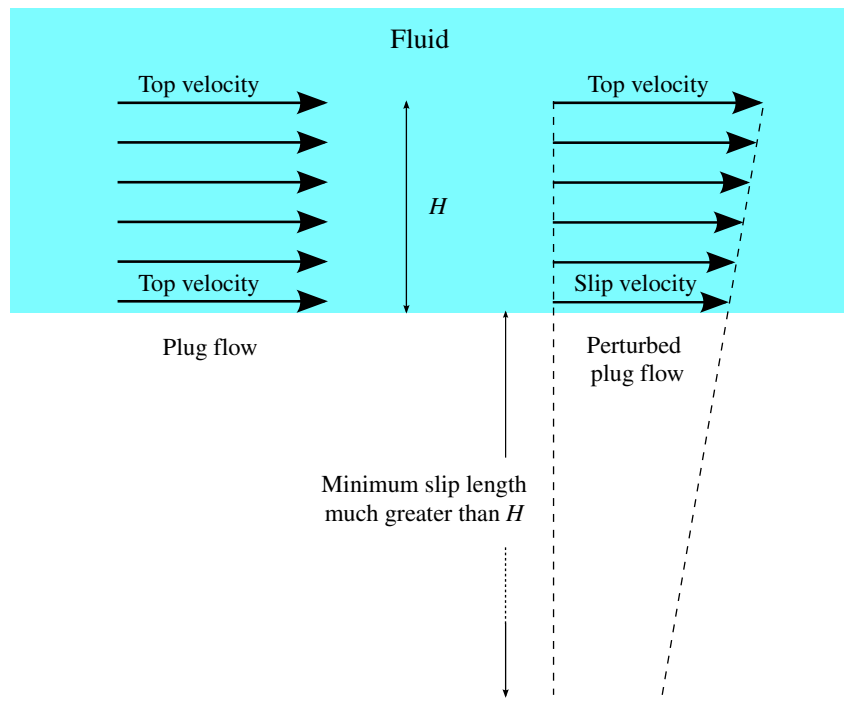

FIgURE 3. Physical illustration of perturbed plug flow. To remain close to plug flow, the slip velocity must be only a small fraction less than the top velocity. Thus, the minimum slip length must be greater than the height of the top velocity, that is, $\delta_{\min } \gg H$.

In nondimensional form, the steady-state Navier-Stokes equations are

$$
\operatorname{Re}[(\hat{u} \cdot \hat{\nabla}) \hat{u}]=-\frac{p_{0} L}{\mu u_{D}} \hat{\nabla} \hat{p}+\hat{\nabla}^{2} \hat{u}, \quad \operatorname{Re}=\frac{u_{D} L}{v} .
$$

In the nanoscale superhydrophobic regime for which $\delta_{\min } \gg L$, with $\delta_{\min } \sim 20 \mathrm{~nm}$, the Reynolds number is negligibly small. With a vanishing left-hand side, the NavierStokes equation reduces to the Stokes equation. Returning to physical units, the fluid motion is now specified by the Stokes equation

$$
\frac{1}{\mu} \nabla p=\nabla^{2} \vec{u}
$$

and (2.1)-(2.5).

The top boundary condition, the driving velocity $u_{D}$, is assumed to be well above the boundary layer, that is, $D \gg H$. (An alternative approach is treat the problem as a boundary layer problem: asymptotic solutions above and below the boundary layer are matched at the boundary layer.)

Perturbed plug flow Formally, the exact solution is assumed to be expressible as a power series in a small parameter, $\epsilon$, with the zeroth-order term identical to plug flow. After appropriate substitution, the boundary conditions are expressions containing $\epsilon$, with the zeroth order yielding plug flow.

The slip length is written as a variation about its minimum

$$
\delta(x, y)=f(x, y) \delta_{\min },
$$


where $f(x, y) \geq 1$ is a dimensionless function of position. The slip boundary condition becomes

$$
\delta_{\min } f(x, y) \frac{\partial}{\partial z} u(x, y, 0)=u(x, y, 0) .
$$

In terms of the small parameter,

$$
f(x, y) L \frac{\partial}{\partial z} u(x, y, 0)=\epsilon u(x, y, 0) .
$$

As $\epsilon \rightarrow 0$, the no-shear condition leading to plug flow is recovered.

The exact velocity solution to (2.1)-(2.6) is expressed as a power series in $\epsilon$,

$$
\vec{u}=\vec{u}_{0}+\epsilon \vec{u}_{1}+O\left(\epsilon^{2}\right),
$$

where

$$
\overrightarrow{u_{0}}+\epsilon \overrightarrow{u_{1}}=\left(u_{0}, v_{0}, w_{0}\right)+\epsilon\left(u_{1}, v_{1}, w_{1}\right)
$$

and the pressure as a perturbation series

$$
p=p_{0}+\epsilon p_{1}+O\left(\epsilon^{2}\right) .
$$

Both (2.7) and (2.8) are inserted into (2.1), (2.2), (2.4)-(2.6), giving, to first order:

$$
\begin{gathered}
\nabla^{2} \vec{u}_{0}+\epsilon \nabla^{2} \vec{u}_{1}=\frac{1}{\mu} \nabla p_{0}+\epsilon \frac{1}{\mu} \nabla p_{1}, \\
\nabla \cdot \vec{u}_{0}+\epsilon \nabla \cdot \vec{u}_{1}=0, \quad u_{0}(x, y, D)+\epsilon u_{1}(x, y, D)=u_{D}, \\
u_{0}(x, y, z)+\epsilon u_{1}(x, y, z)=u_{0}(x+L, y, z)+\epsilon u_{1}(x+L, y, z), \\
f(x, y) L \frac{\partial}{\partial z} u_{0}(x, y, 0)+\epsilon f(x, y) L \frac{\partial}{\partial z} u_{1}(x, y, 0)=\epsilon u_{0}(x, y, 0) .
\end{gathered}
$$

Zeroth order Setting $\epsilon$ to zero in system (2.9) yields the zeroth-order equations

$$
\begin{gathered}
\nabla^{2} \vec{u}_{0}=\frac{1}{\mu} \nabla p_{0}, \quad \nabla \cdot \vec{u}_{0}=0, \quad u_{0}(x, y, D)=u_{D}, \\
u_{0}(x, y, z)=u_{0}(x+L, y, z), \quad f(x, y) L \frac{\partial}{\partial z} u_{0}(x, y, 0)=0 .
\end{gathered}
$$

As defined, the solution is plug flow: $u_{0}(x, y, z)=u_{D}, x$ velocity constant throughout the channel.

First order Cancelling zeroth-order terms and dividing by $\epsilon$ yields the first-order equations

$$
\begin{gathered}
\nabla^{2} \vec{u}_{1}=\frac{1}{\mu} \nabla p_{1}, \\
\nabla \cdot \vec{u}_{1}=0, \quad w_{1}(x, y, 0)=0, \quad u_{1}(x, y, D)=0, \\
u_{1}(x, y, z)=u_{1}(x+L, y, z), \quad f(x, y) L \frac{\partial}{\partial z} u_{1}(x, y, 0)=u_{0}(x, y, 0)=u_{D} .
\end{gathered}
$$


We eliminate pressure using the vorticity equation. Taking the curl of the Stokes equation (2.10) gives

$$
\nabla \times \nabla^{2} \vec{u}_{1}=\nabla \times \frac{1}{\mu} \nabla p_{1} .
$$

The right-hand side of (2.11) is identically zero. The remaining equation $\nabla \times$ $\nabla^{2} \vec{u}_{1}=0$ gives us three partial differential equations (PDEs)

$$
\begin{aligned}
& \frac{\partial^{3} w_{1}}{\partial y \partial x^{2}}+\frac{\partial^{3} w_{1}}{\partial y^{3}}+\frac{\partial^{3} w_{1}}{\partial y \partial z^{2}}=\frac{\partial^{3} v_{1}}{\partial z \partial x^{2}}+\frac{\partial^{3} v_{1}}{\partial z \partial y^{2}}+\frac{\partial^{3} v_{1}}{\partial z^{3}} \\
& \frac{\partial^{3} u_{1}}{\partial z \partial x^{2}}+\frac{\partial^{3} u_{1}}{\partial z \partial y^{2}}+\frac{\partial^{3} u_{1}}{\partial z^{3}}=\frac{\partial^{3} w_{1}}{\partial x^{3}}+\frac{\partial^{3} w_{1}}{\partial x \partial y^{2}}+\frac{\partial^{3} w_{1}}{\partial x \partial z^{2}}, \\
& \frac{\partial^{3} u_{1}}{\partial y \partial x^{2}}+\frac{\partial^{3} u_{1}}{\partial y^{3}}+\frac{\partial^{3} u_{1}}{\partial y \partial z^{3}}=\frac{\partial^{3} v_{1}}{\partial x^{3}}+\frac{\partial^{3} v_{1}}{\partial x \partial y^{2}}+\frac{\partial^{3} v_{1}}{\partial x \partial z^{2}}
\end{aligned}
$$

In order to capitalize on our boundary conditions, we want expressions in $u_{1}$ and $w_{1}$. Equation (2.12) can be used directly. Equation (2.13) is differentiated with respect to $y$, enabling a substitution for $\partial v_{1} / \partial y$ using the continuity equation

$$
\frac{\partial v_{1}}{\partial y}=-\frac{\partial u_{1}}{\partial x}-\frac{\partial w_{1}}{\partial z}
$$

This yields two PDEs in $u_{1}$ and $w_{1}$,

$$
\begin{gathered}
\frac{\partial^{3} u_{1}}{\partial x^{2} \partial z}+\frac{\partial^{3} u_{1}}{\partial y^{2} \partial z}+\frac{\partial^{3} u_{1}}{\partial z^{3}}=\frac{\partial^{3} w_{1}}{\partial x^{3}}+\frac{\partial^{3} w_{1}}{\partial x \partial y^{2}}+\frac{\partial^{3} w_{1}}{\partial x \partial z^{2}} \\
\frac{\partial^{4} u_{1}}{\partial x^{4}}+2 \frac{\partial^{4} u_{1}}{\partial x^{2} \partial y^{2}}+\frac{\partial^{4} u_{1}}{\partial x^{2} \partial z^{2}}+\frac{\partial^{4} u_{1}}{\partial y^{4}}+\frac{\partial^{4} u_{1}}{\partial y^{2} \partial z^{2}}=-\frac{\partial^{4} w_{1}}{\partial x^{3} \partial z}-\frac{\partial^{4} w_{1}}{\partial x \partial y^{2} \partial z}-\frac{\partial^{4} w_{1}}{\partial x \partial z^{3}} .
\end{gathered}
$$

Because the flow is periodic, it is natural to write $u_{1}$ as a Fourier series

$$
u_{1}(x, y, z)=\sum_{\vec{k}}^{\infty} U_{\vec{k}}(z) \exp (i \vec{k} \cdot \vec{r})
$$

where the wave vector $\vec{k}$ is a reciprocal lattice vector defined by integers $p, q$,

$$
\vec{k}=(m, n)=(2 \pi p, 2 \pi q), \quad k^{2}=m^{2}+n^{2},
$$

and the Fourier coefficient is

$$
U_{\vec{k}}(z)=\frac{1}{L^{2}} \int_{0}^{L} \int_{0}^{L} u_{1}(x, y, z) \exp (i \vec{k} \cdot \vec{r}) d x d y .
$$


Similarly for $w_{1}$,

$$
w_{1}(x, y, z)=\sum_{\vec{k}}^{\infty} W_{\vec{k}}(z) \exp (i \vec{k} \cdot \vec{r}) .
$$

The two velocity expressions (2.16) and (2.17) are inserted into (2.14) and (2.15). The resulting expressions are true for arbitrary $\vec{r}=(x, y)$, so it is required that, for all $k$,

$$
\begin{gathered}
\frac{d^{3}}{d z^{3}} U(z)-k^{2} \frac{d}{d z} U(z)=i\left(\frac{d^{2}}{d z^{2}} W(z)-k^{2} W(z)\right) m \\
k^{2} \frac{d^{2}}{d z^{2}} U(z)-k^{4} U(z)=i\left(\frac{d^{3}}{d z^{3}} W(z)-k^{2} \frac{d}{d z} W(z)\right) m .
\end{gathered}
$$

(The variables $k$ and $m$ are of course not independent.) After differentiating (2.19) with respect to $z$, and multiplying (2.18) by $k^{2}$, the two equations may be combined, giving an ordinary differential equation (ODE) in $W(z)$ alone (defined for nonzero $k$ ),

$$
\frac{d^{4}}{d z^{4}} W(z)-2 k^{2} \frac{d^{2}}{d z^{2}} W(z)+k^{4} W(z)=0,
$$

whose solution is

$$
W(z)=\left(P_{\vec{k}}+Q_{\vec{k}} z\right) e^{-k z}+\left(R_{\vec{k}}+S_{\vec{k}} z\right) e^{k z} .
$$

Now, the $z$ velocity vanishes at the top of the fluid where $z=D$, that is, $W(D)=0$. Furthermore, $D$ is arbitrarily large. For large $D$, the $e^{-k D}$ term is negligible, but the $e^{k D}$ term is singular as $D \rightarrow \infty$. Thus, the top boundary condition holds if and only if $R_{\vec{k}}=S_{\vec{k}}=0$. We are left with $W(z)=\left(P_{\vec{k}}+Q_{\vec{k}} z\right) e^{-k z}$. The $z$ velocity vanishes at the bottom surface also, hence $W(0)=P_{\vec{k}}=0$, leaving just

$$
W(z)=Q_{\vec{k}} z e^{-k z} .
$$

Equation (2.20) may be inserted back into (2.19) giving an ODE in $U(z)$,

$$
k^{2} \frac{d^{2}}{d z^{2}} U(z)-k^{4} U(z)=i Q_{\vec{k}} m k^{2} e^{-k z}
$$

whose solution is

$$
U_{\vec{k}}(z)=\left(P_{\vec{k}}+i Q_{\vec{k}} \frac{m}{k^{2}}\right) e^{-k z}+B_{\vec{k}} e^{k z} .
$$

This is defined for nonzero $k$. For $k=0, W(z)$ is not defined, and from (2.18), $U(z)$ satisfies $d^{3} U(z) / d z^{3}=0$, whose solution is $U_{0}=A_{0}+B_{0} z+C_{0} z^{2}$, so that

$$
u_{1}(x, y, z)=A_{0}+B_{0} z+C_{0} z^{2}+\sum_{\vec{k} \neq \overrightarrow{0}}\left(A_{\vec{k}} e^{-k z}+B_{\vec{k}} e^{k z}\right) e^{i \vec{k} \cdot \vec{r}}
$$


where $A_{\vec{k}}=P_{\vec{k}}+i Q_{\vec{k}} m / k^{2}$. Inserting this solution into the $x$ component of the Stokes equation

$$
\frac{\partial^{2}}{\partial x^{2}} u_{1}+\frac{\partial^{2}}{\partial y^{2}} u_{1}+\frac{\partial^{2}}{\partial z^{2}} u_{1}=\frac{1}{\mu} \frac{\partial}{\partial x} p_{1}
$$

gives

$$
2 C_{0}+\sum_{\vec{k} \neq \overrightarrow{0}}\left(A_{\vec{k}} e^{-k z}+B_{\vec{k}} e^{k z}\right)\left(k^{2}-k^{2}\right) e^{i \vec{k} \cdot \vec{r}}=\frac{1}{\mu} \frac{\partial}{\partial x} p_{1},
$$

so that

$$
2 C_{0}=\frac{1}{\mu} \frac{\partial}{\partial x} p_{1} .
$$

The flow is shear-driven only, thus $p$ is a periodic function of $x$. Therefore, by integrating (2.22) over one period of $x$, we eliminate the right-hand side.

$$
2 C_{0} L=\frac{1}{\mu}\left[p_{1}(L, y, z)-p_{1}(0, y, z)\right]=0 .
$$

The quadratic coefficient $C_{0}$ in (2.21) vanishes. We now have

$$
u_{1}(x, y, z)=A_{0}+B_{0} z+\sum_{\vec{k} \neq \overrightarrow{0}}\left(A_{\vec{k}} e^{-k z}+B_{\vec{k}} e^{k z}\right) e^{i \vec{k} \cdot \vec{r}} .
$$

The top boundary condition is

$$
u_{1}(x, y, D)=A_{0}+B_{0} D+\sum_{\vec{k} \neq 0}\left(A_{\vec{k}} e^{-k D}+B_{\vec{k}} e^{k D}\right) e^{i \vec{k} \cdot \vec{r}}=0 .
$$

Now, $D$ is of course arbitrary, and is assumed to be sufficiently large that perturbations due to the surface have died away. Thus, the periodic part (sum) of $u_{1}$, and the mean part (constant), must both vanish at $z=D$. The $e^{-k D}$ term is negligible, but the $e^{k D}$ term is singular as $D \rightarrow \infty$, so for all $k$ the coefficient $B_{\vec{k}}$ is zero. The constant term must be $A_{0}=-B_{0} D$, and we are left with

$$
u_{1}(x, y, z)=B_{0}(z-D)+\sum_{\vec{k} \neq 0}\left(A_{\vec{k}} e^{-k z}\right) e^{i \vec{k} \cdot \vec{r}} .
$$

To find $B_{0}$, we make use of the first-order slip condition

$$
\frac{\partial}{\partial z} u_{1}(x, y, 0)=\frac{u_{D}}{f(x, y) L} .
$$

Differentiating the definition of zeroth-Fourier coefficient gives the relation

$$
\frac{d}{d z}\left(B_{0} z-B_{0} D\right)=\frac{1}{L^{2}} \int_{0}^{L} \int_{0}^{L} \frac{\partial}{\partial z} u_{1}(x, y, z) d x d y .
$$


Evaluating at $z=0$ gives

$$
\begin{aligned}
B_{0} & =\frac{1}{L^{2}} \int_{0}^{L} \int_{0}^{L} \frac{\partial}{\partial z} u_{1}(x, y, 0) d x d y \\
& =\frac{1}{L^{2}} \int_{0}^{L} \int_{0}^{L} \frac{u_{D}}{f(x, y) L} d x d y=\frac{u_{D}}{L}\left\langle\frac{1}{f(x, y)}\right\rangle
\end{aligned}
$$

so our $x$-velocity pertubative solution is

$$
u(x, y, z)=u_{s}+\epsilon \frac{u_{D}}{L}\left\langle\frac{1}{f(x, y)}\right\rangle(z-D)+\epsilon \sum_{\vec{k} \neq 0}\left(A_{\vec{k}} e^{-k z}\right) e^{i \vec{k} \cdot \vec{r}}
$$

The small parameter $\epsilon$ may be folded into the area average $f(x, y)$ term

$$
\epsilon \frac{u_{D}}{L}\left\langle\frac{1}{f(x, y)}\right\rangle=\frac{L}{\delta_{\min }} \frac{u_{D}}{L}\left\langle\frac{1}{f(x, y)}\right\rangle=u_{D}\left\langle\frac{1}{\delta(x, y)}\right\rangle .
$$

Thus, our final velocity equation is

$$
u(x, y, z)=u_{D}+u_{D}\left\langle\frac{1}{\delta(x, y)}\right\rangle(z-D)+\epsilon \sum_{\vec{k} \neq \overrightarrow{0}}\left(A_{\vec{k}} e^{-k z}\right) e^{i \vec{k} \cdot \vec{r}}
$$

Effective slip length At the driving height $D$ above the surface, perturbations due to the mixed-slip surface have died away, and the fluid velocity is constant. The shear rate at $D$ will determine an effective slip length, via the Navier slip condition:

$$
\begin{gathered}
\delta_{\mathrm{eff}} \frac{\partial}{\partial z} u(x, y, D)=u(x, y, D) \\
\delta_{\mathrm{eff}}\left[u_{D}\left\langle\frac{1}{\delta(x, y)}\right\rangle+\epsilon \sum\left(-k A_{\vec{k}} e^{-k D}\right) e^{i \vec{k} \cdot \vec{r}}\right]=u_{D}
\end{gathered}
$$

Now, the sum term is negligible at $D$, so we are left with:

$$
\delta_{\mathrm{eff}}=\left\langle\frac{1}{\delta(x, y)}\right\rangle^{-1}
$$

This is the key result of this paper.

Perturbed Couette flow A similar analysis perturbing Couette flow yields an effective slip length given by the simple area average of slip lengths,

$$
\delta_{\text {eff }}=\langle\delta(x, y)\rangle
$$




\section{Discussion}

Equation (2.23) is valid for $\delta_{\min } \gg H$, that is, for a nanoscale superhydrophobic surface, where the perturbed boundary layer is sufficiently thin that flow within the boundary layer can be considered plug-like. The effective slip length determines the flow for the rest of the fluid, which need not be plug-like.

For a binary gas-solid surface satisfying $\delta_{s} \gg H$, with $\phi$ the area fraction of the solid, the relation is

$$
\delta_{\text {eff }}=\left[\phi \frac{1}{\delta_{s}}+(1-\phi) \frac{1}{\delta_{g}}\right]^{-1} .
$$

Thus, slip lengths add like (area-weighted) capacitors in series, or resistors in parallel.

Other formulations include

$$
\delta_{\mathrm{eff}}=\frac{\delta_{g}}{\phi\left(\left(\delta_{g} / \delta_{s}\right)-1\right)+1}=\frac{\delta_{s}}{\phi\left(1-\left(\delta_{s} / \delta_{g}\right)\right)+\left(\delta_{s} / \delta_{g}\right)},
$$

which illustrate the basic form of inverse- $\phi$-dependence $\delta_{\mathrm{eff}}=1 /(a \phi+b)$.

In practice, the slip length over gas may be very large. As $\delta_{g} \rightarrow \infty$, the relation reduces to $\delta_{\text {eff }}=\delta_{s} / \phi$. In the case of alternating strips of period $L$, with $a$ being the length of the solid part, and a divergent gas slip length, we expect $\delta_{\text {eff }}=L \delta_{s} / a$. If $a$ were held fixed, and $L$ varied, then obviously a linear dependence of $\delta_{\text {eff }}$ on $L$ would result. This could hold in the case of carbon nanoforests, where the density is varied, but nanotube diameter is unchanged.

Using the length scale $L$ in the perturbation parameter has the advantage that it can in principle be determined unambiguously. As noted, earlier work [10] has shown that $H \sim L$. For fixed $L$, it is expected that $H$ peaks at $\phi \approx 0.5$. Now, in some regimes with small $\phi$, it will be the case that $L>H$. In those cases, an analysis assuming $\delta_{\min } \gg L$ will still give valid results even though in fact $\delta_{\min } \sim L$, simply because it is still true that $\delta_{\min } \gg H$. This has been observed: our previous numerical calculations in [11] agree with (3.1) for $\delta_{\min }$ values down to $\delta_{\min } \sim L$, and at $\delta_{\min }=0.1 L$ the discrepancy peaks at $\phi \approx 0.5$, as expected. Cottin-Bizonne et al. compute values that agree with (3.1) down to $\delta_{\min }=0.1 L$ [6].

One limitation of our work is the assumption that the interface is a flat plane. In most real situations, some degree of curvature will be present. On a superhydrophobic surface, pressure pushes the liquid down into the gas regions, increasing the surface area of the liquid-gas interface. Nanobubbles on a hydrophobic surface obviously have convex surfaces. Accounting for these effects would be a useful extension of the work. A superhydrophobic microgrooved surface was studied by Sbragaglia and Prosperetti, who used a perturbative approach to quantify the change in slip length caused by curvature [18].

\section{Conclusion}

We have shown that for flow that is close to plug flow, over an idealized flat surface, patterned with doubly-periodic variation in intrinsic slip length, with minimum slip 
length not less than the pattern period, the effective slip length is given by

$$
\delta_{\text {eff }}=\left\langle\frac{1}{\delta(x, y)}\right\rangle^{-1},
$$

which, for a binary surface such as the fluid interface on a nanoscale superhydrophobic surface, or a hydrophobic surface decorated with nanobubbles, reduces to

$$
\delta_{\mathrm{eff}}=\left[\phi \frac{1}{\delta_{s}}+(1-\phi) \frac{1}{\delta_{g}}\right]^{-1},
$$

where $\delta_{s}, \delta_{g}$ are the intrinsic slip lengths of the solid and gaseous regions, respectively, and $\phi$ is the area fraction of the solid region.

\section{Acknowledgement}

N. J. Lund wishes to thank New Zealand Institute for Mathematics and its Applications for scholarship funding.

\section{References}

[1] J.-L. Barrat and L. Bocquet, "Influence of wetting properties on hydrodynamic boundary conditions at a fluid/solid interface", Faraday Discuss. 112 (1999) 119-127.

[2] J.-L. Barrat and L. Bocquet, "Large slip effect at a nonwetting fluid-solid interface", Phys. Rev. Lett. 82 (1999) 4671-4674.

[3] J. Baudry, E. Charlaix, A. Tonck and D. Mazuyer, "Experimental evidence for a large slip effect at a nonwetting fluid-solid interface", Langmuir 17 (2001) 5232-5236.

[4] C.-H. Choi and C.-J. Kim, "Large slip of aqueous liquid flow over a nanoengineered superhydrophobic surface", Phys. Rev. Lett. 96 (2006) 066001.

[5] N. V. Churaev, V. D. Sobolev and A. N. Somov, "Slippage of liquids over lyophobic solid surfaces", J. Colloid Interface Sci. 97 (1984) 574-581.

[6] C. Cottin-Bizonne, C. Barentin, E. Charlaix, L. Bocquet and J.-L. Barrat, "Dynamics of simple liquids at heterogeneous surfaces: molecular-dynamics simulations and hydrodynamics description", Eur. Phys. J. E 15 (2004) 427-438.

[7] C. Cottin-Bizonne, J.-L. Barrat, L. Bocquet and E. Charlaix, "Low-friction flows of liquid at nanopatterned interfaces", Nature Mater. 2 (2003) 237-240.

[8] V. S. J. Craig, C. Neto and D. R. M. Williams, "Shear-dependent boundary slip in an aqueous Newtonian liquid", Phys. Rev. Lett. 87 (2001) 054504.

[9] P. G. de Gennes, "On fluid/wall slippage”, Langmuir 18 (2002) 3413-3414.

[10] S. C. Hendy, M. Jasperse and J. Burnell, "Effect of patterned slip on micro- and nanofluidic flows", Phys. Rev. E 72 (2005) 016303.

[11] S. C. Hendy and N. J. Lund, "Effective slip boundary conditions for flows over nanoscale chemical heterogeneities", Phys. Rev. E 76 (2007) 066313.

[12] P. Joseph, C. Cottin-Bizonne, J.-M. Benot, C. Ybert, C. Journet, P. Tabeling and L. Bocquet, "Slippage of water past superhydrophobic carbon nanotube forests in microchannels", Phys. Rev. Lett. 97 (2006) 156104.

[13] E. Lauga and H. A. Stone, "Effective slip in pressure-driven Stokes flow", J. Fluid Mech. 489 (2003) 55-77.

[14] C. Neinhuis and W. Barthlott, "Characterization and distribution of water-repellent, self-cleaning plant surfaces", Ann. Bot. 79 (1997) 667-677. 
[15] T. Onda, S. Shibuichi, N. Satoh and K. Tsujii, "Super-water-repellent fractal surfaces", Langmuir 12 (1996) 2125-2127.

[16] J. R. Philip, "Flows satisfying mixed no-slip and no-shear conditions", Z. Angew. Math. Phys. 23 (1972) 353-372.

[17] D. Qur, "Fakir droplets", Nature Mater. 1 (2002) 14-15.

[18] M. Sbragaglia and A. Prosperetti, "A note of the effective slip properties for microchannel flows with ultrahydrophobic surfaces", Phys. Fluids 19 (2007) 043603.

[19] E. Schnell, "Slippage of water over nonwettable surfaces", J. Appl. Phys. 27 (1956) 1149-1152.

[20] P. A. Thompson and M. O. Robbins, "Shear flow near solids: epitaxial order and flow boundary conditions", Phys. Rev. A 41 (1990) 6830-6837.

[21] O. I. Vinogradova, "Slippage of water over hydrophobic surfaces", Int. J. Miner. Process. 56 (1999) $31-60$.

[22] X. H. Zhang, A. Quinn and W. A. Ducker, "Nanobubbles at the interface between water and a hydrophobic solid", Langmuir 24 (2008) 4756-4764.

[23] Y. Zhu and S. Granick, "Rate-dependent slip of Newtonian liquid at smooth interfaces", Phys. Rev. Lett. 87 (2001) 096105.

[24] Y. Zhu and S. Granick, "Limits of the hydrodynamic no-slip boundary condition", Phys. Rev. Lett. 88 (2002) 106102. 